\title{
Dynamic DNA methylation
}

\section{DOI:}

$10.1038 / \mathrm{nrn} 2133$

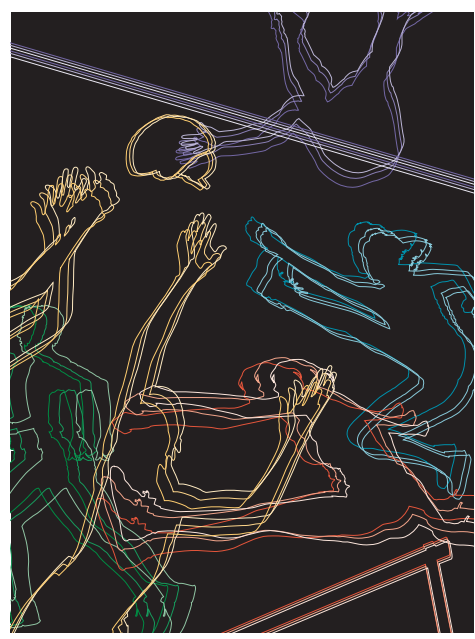

During development, posttranslational modifications to DNA and histones allow mitotic cells to perpetuate phenotype-specific gene expression patterns. It has been suggested that similar epigenetic mechanisms might contribute to the formation or storage of long-term memories in the adult. Miller and Sweatt now show that covalent DNA modifications are involved in memory formation in the hippocampus.

Methylation of DNA by DNA (cytosine-5') methyltransferases (DNMTs) induces long-term transcriptional silencing during development. DNMTs are also active in the adult brain, and misregulated DNA methylation has been linked to several neurological disorders; however, it is not known how DNA methylation contributes to normal brain function. The authors therefore investigated the role of this process in associative memory formation in the hippocampus of adult rats.

The authors used a contextual fear-conditioning protocol in which the environment of the training cage was associated with delivery of a footshock. They demonstrated that DNMT mRNA levels were increased 30 minutes after the training session, suggesting that DNMT activity is regulated in response to stimuli that evoke memory formation. Treatment with DNMT inhibitors shortly after training blocked memory consolidation, resulting in reduced freezing behaviour when the animals entered the training cage in a subsequent session. This effect was transient: 24 hours after treatment, the animals were able to form normal associative memories.

To determine which genes might be regulated by DNMT activity during memory formation, the authors investigated the methylation status of two genes: protein phosphatase 1 (Pp1), which suppresses memory formation, and reelin, which promotes synaptic plasticity and memory formation. They showed that the levels of methylated $P p 1$ increased 1 hour after fear conditioning, with a corresponding decrease in $P p 1$ transcription. By contrast, levels of methylated reelin decreased in response to fear-conditioning, accompanied by increased levels of unmethylated reelin and reelin transcription. Unlike the long-lasting changes in DNA methylation typically observed during development, the altered gene expression observed after fear conditioning reverted to normal levels within 24 hours.
Consistent with their effect on memory formation, treatment with DNMT inhibitors attenuated the fear-conditioning-induced methylation of $P p 1$. Interestingly, the effects of inhibition of DNMT and fearconditioning on the demethylation of reelin were additive, suggesting that one or more DNA demethylases also contribute to the effects of fearconditioning on the expression of this gene. DNMT inhibitors were unable to alter gene transcription in the absence of fear conditioning, indicating that DNA methylation might work together with additional activity-dependent mechanisms to regulate the expression of these genes.

This study shows for the first time that DNA methylation and demethylation are dynamic ongoing processes in the adult brain. The modifications in response to fear-conditioning were unexpectedly short-lived, pointing to a role for these mechanisms in memory formation rather than long-term storage. This study opens up numerous directions for future research, including investigating the mechanisms regulating DNMT and demethylase activity and determining whether similar mechanisms are at work in other regions of the brain and in the formation of other types of memory.

Katherine Whalley

ORIGINAL RESEARCH PAPER Miller, C. A. \&

Sweatt, D.W. Covalent modification of DNA regulates memory formation. Neuron 53, 857-869 (2007)

FURTHER READING Levenson, J. M. \&

Sweatt, D.W. Epigenetic mechanisms in memory formation. Nature Rev. Neurosci. 6, 108-118 (2005) 